\title{
Non-Specific Vaccination Exerts Anti-Tumor Effect through CD11c (+) Cells Stimulation in a Breast Cancer Model
}

\section{Vanessa Elizabeth De Gregorio', Silvia Carino', Juan Carlos Valdez², Silvio Eugenio Castillo ${ }^{3}$ and Alejandra de Moreno de LeBlanc ${ }^{4 *}$}

\author{
${ }^{1}$ Laboratorio de Anatomía Patológica, Facultad de Odontología, Universidad Nacional de Tucumán, Argentina \\ ${ }^{2}$ Cátedra de Inmunología, Facultad de Bioquimíca, Universidad Nacional de Tucumán, Argentina \\ ${ }^{3}$ Facultad de Ciencias Naturales, Universidad Nacional de Tucumán, Argentina \\ ${ }^{4}$ Centro de Referencia para Lactobacilos (CERELA-CONICET), Argentina
}

*Corresponding author: Alejandra de Moreno de LeBlanc, Centro de Referencia para Lactobacilos (CERELA-CONICET), Chacabuco 145, San Miguel de Tucumán, Tucumán, Argentina, E-mail: demoreno@cerela.org.ar; demorenoalejandra@gmail.com

\begin{abstract}
Background: Immunotherapy by using non-specific vaccines has proven to be effective in experimental animal models and also in patients suffering cancer. In the present work, the effectiveness of this immunotherapy was evaluated using a murine breast cancer model and a polymicrobial vaccine.

Methodology/Principal: Mice bearing injected with tumor cells from the spontaneous syngeneic mammary adeno carcinoma M3 were used as breast cancer model. The immune adjuvant effect of the vaccine was analyzed in comparison or in the presence of doxorubicin. Tumor volume was calculated. Tumor, spleen and lymph nodes were processed for histological observations and to determinate the percentage of CD11c (+) cells.

Results: Mice treated with the vaccine or with doxorubicin decreased tumor growth, with less tumor cell invasion. Tumor tissues showed decreased necrosis and nuclear areas in mice that received any of the treatments under study, compared with the control group. The benefits of vaccination were associated to stimulation of the immune response. More hyperplasia of the red and white pulp, and increased marginal megakaryocytes were observed in the spleens from mice treated with the vaccine. CD11c $(+)$ cells did not increase in tumor draining nodes by the immune stimulating effect of the vaccine; however, this treatment increased CD11c $(+)$ cells in the spleen and tumor.

Conclusions: A non-specific vaccine used as immunotherapy can be used to prolong an equilibrium phase in the tumor growth, similar to the doxorubicin treatment used in the model, although by different mechanisms. Non synergic effect was observed in the group treated with both vaccine and doxorubicin (low dose). Future studies should be necessary to evaluate the correct combination of vaccination and cytotoxic drug can be used for this purpose and to achieve increased activated dendritic cells in the tumor draining lymph nodes.
\end{abstract}

\section{Keywords}

Breast cancer, Immune response, Vaccine, Dendritic cells

\section{Introduction}

Cancer is still one of the most common causes of mortality and morbidity throughout the world [1]. The possibilities of successful treatments accompanied or not by recurrence depend of the tumor type and the time of tumor growth in which it was discovered. The main treatments for cancer are surgery, chemotherapeutic agents and ionizing radiation to kill the tumor mass. These therapies have provided great benefits; however, the incidence of tumor recurrence is a major problem resulting from the development of mechanisms of resistance against the drugs and/or radiation by a portion of the tumor cells. This has led to the search for additional therapies that cause less secondary effects. One such strategy is the use of treatments designed to take advantage of the specificity of the immune system to recognize and kill tumor cells.

The concept that the immune system can respond against tumor development is now well recognized [2]. Data from animal models, together with data from human patients, indicate that the immune system is critical in the detection and eradication of tumor. Patients with congenital or acquired immunodeficiency, as well as patients undergoing immunosuppressive therapies have higher incidence of tumors compared to immuno competent individuals [3]. However, it is important to highlight that radiotherapy and chemotherapy, well established treatments of choice against cancer, are highly immunosuppressive.

A better understanding of the molecular interactions between tumors and the host immune system is essential for the development of new approaches for the simultaneous control of tumor escape routes and activation of antitumor immune responses. The combination of chemotherapeutic drugs in addition to immunotherapy with nonspecific anti-tumor vaccines can cause a beneficial immune response and increase the effectiveness of vaccines [4]. There are studies that have shown that non-specific vaccines can act to stimulate the host immune response against tumors, being William Coley the first who successfully used this methodology [5]. Since the descriptions

Citation: Gregorio VED, Carino S, Valdez JC, Castillo SE, LeBlanc AM (2016) Non-Specific Vaccination Exerts Anti-Tumor Effect through CD11c (+) Cells Stimulation in a Breast Cancer 
Table 1: Vaccine composition.

\begin{tabular}{|l|l|l|l|l|l|}
\hline Microorganism & Vial N`1 & Vial N² & Vial N³ & Vial N4 & Vial N$^{*} \mathbf{5}$ \\
\hline Streptococcus pneumoniae & 100 & 200 & 300 & 400 & 600 \\
\hline Haemophilus influenza & 50 & 100 & 150 & 200 & 300 \\
\hline Klebsiella pneumoniae & 50 & 100 & 150 & 200 & 300 \\
\hline Micrococcus sp. (respiratory) & 50 & 100 & 150 & 200 & 200 \\
\hline Branhamellacatarrhalis & 50 & 150 & 150 & 200 & 200 \\
\hline Streptococcus sp. (respiratory) & 100 & 300 & 300 & 400 & 400 \\
\hline Excipients ${ }^{\dagger}$ & & & & & \\
\hline Povidone & 8.0 & 8.0 & 8.0 & 8.0 & 8.0 \\
\hline Sodium chloride & 8.5 & 8.5 & 8.5 & 8.5 & 8.5 \\
\hline Mannitol & 50.0 & 50.0 & 50.0 & 50.0 & 50.0 \\
\hline
\end{tabular}

\#The count of microorganisms in each vial is expressed $\times 10^{6}$.

tThe concentration of excipients in each vial is expressed in $\mathrm{mg}$.

by Coley, different bacterial agents have been evaluated as immune adjuvants. Non-specific immune stimulation using Bacillus CalmetteGuerin (BCG) was reported [6], especially against bladder cancer [7]. The vaccine was injected into the bladder through a catheter and BCG attracted the host's immune cells to the bladder in order to attack the cancer cells. However, recent reports have shown the possibility (although low) of widespread BCG infection following intra vesicle treatment, and the need for vigilance in patients with any susceptibility or long term use of high doses of BCG [8-10].

Following this tendency to stimulate non-specifically the host immune system to attack cancer cells, the aim of the present work was to evaluate the immunotherapeutic effect of a polymicrobial vaccine, combined with cytostatic agents, using a scheme of multiple vaccinations in a murine breast cancer model. The chemotherapy and immune stimulating treatment were administered after tumor cell inoculation, determining its effectiveness by measuring tumor volume and its impact on the immune system by studying the histological architecture of the lymphoid organs and infiltrating dendritic cells in the tumor, lymph nodes and spleen.

\section{Material and Methods}

\section{Animals and tumour induction}

Six-week-old female BALB/c mice weighing $22 \pm 2 \mathrm{~g}$ were obtained from the random-bred colony maintained at Facultad de Medicina, Universidad Nacional de Tucuman (San Miguel de Tucumán, Argentina). The spontaneous syngeneic mammary adenocarcinoma M3 was acquired from the Instituto de Oncología A.H. Roffo, Universidad de Buenos Aires (Buenos Aires, Argentina), which previously characterized it [11]. The tumor line was maintained by in vivo passage of the tumor cells to six syngeneic female mice every 15 days.

For the assay, one tumor was removed aseptically by surgery and placed into a sterile tube containing RPMI-1640 medium (Sigma, St Louis, MO, USA). It was then disaggregated mechanically with scissors and exposed to enzyme digestion. All enzymes were purchased from Sigma (St Louis, MO, USA). Tumours pieces were digested with protease (Pronase E) $0.05 \%$ for $15 \mathrm{~min}$ at $37^{\circ} \mathrm{C}$, stirring slightly with a magnetic agitator. The cell suspension was then washed with RPMI- 1640 and treated with DNase $0.001 \%$ followed by centrifugation at low speed $(800 \mathrm{~g})$ to harvest the tumour cells. The pellet was suspended with RPMI 1640 containing $10 \%$ foetal bovine serum (FBS), and the concentration of cells was adjusted at $2 \pm 1 \times 10^{6}$ cell $/ \mathrm{ml}$, by counting in a Neubauer chamber with a solution of trypan blue (to take into account only the number of living cells). Each mouse was injected with $100 \mu \mathrm{l}$ of this cell suspension (approximately $1 \times 10^{5}$ cells per mouse) subcutaneously in the lower right mammary gland.

All animals received balanced chow diet ad libitum and were maintained in a room with a $12 \mathrm{~h}$ light/dark cycle at $18 \pm 2{ }^{\circ} \mathrm{C}$. All experiments comply with the current laws of Argentina. All procedures performed with animals were performed according to bioethical standards established for handling (regulation 6344/96, National Administration of Drugs, Food and Medical Technology -ANMAT) on requirements for animal facilities.

\section{Vaccine and cytostatic agent}

The vaccine used was Vacuna Liofilizada Casasco ${ }^{\circ}$ (Casasco Laboratory, Buenos Aires, Argentina) which is purchased lyophilized in 5 vials, each one with increasing amounts of microorganisms (Table 1). The content of each vial was resuspended in $1 \mathrm{ml}$ of diluents (thimerosal $0.8 \mathrm{mg}$ and distilled water, provided with the vaccine). The vaccine is indicated for prophylaxis of ENT (ear, nose and throat - respiratory) and broncho pulmonary infections. It also contains glycolipid from Escherichia coli that increases circulating properdin (nonspecific immunity) for more than 7 days. Doxorubicin (Doxocris, Laboratory Kampel Martian S.A., Buenos Aires Argentina) was used as cytostatic agent.

\section{Experimental protocol and measurement of tumor growth}

Twenty eight mice were injected with the tumor cells as was explained above and divided into 4 groups (7 mice per group): Mice without treatment (Control group); mice treated with the vaccine (Vaccine group); mice treated with the cytostatic agent (Doxo group), and mice treated with both the vaccine and the cytostatic agent (Vaccine-Doxo group).

For the mice in the Vaccine group, the vaccine (vial 1) was injected intramuscularly $(0.1 \mathrm{ml}) 3$ days after the inoculation of tumor cells. This treatment was followed by 4 injections of $0.1 \mathrm{ml}$ each (days 6, 9, 13 and 17 post tumor cells) with increasing amounts of microorganisms using the vials $2,3,4$ and 5 , respectively.

For Doxo group, doxorubicin treatment consisted of 2 intravenous injections (in the tail vein) of $0.1 \mathrm{ml}$ that contained 150 $\mu \mathrm{g}$ of the drug. The injections were performed 3 and 14 days post inoculation of tumor cells.

For Vaccine-Doxo group, a lower dose of doxorubicin $(30 \mu \mathrm{g})$ was used following the same protocol of 2 injections described above. The vaccination was similar to the Vaccine group, but the first injection was 4 days after the inoculation of tumour cells and the other injections were on days 7, 11, 17 (4 injections in total, without the vaccine from vial $\mathrm{N}^{\circ} 5$ ). The first application of the vaccine was one day after doxorubicin (day 4), and the last inoculation was 6 days after the $3^{\text {rd }}$ one because the second doxorubicin injection was between $3^{\text {rd }}$ and $4^{\text {th }}$ vaccine injection). This protocol was followed to ensure a lower concentration of this cytostatic drug in circulation, and in this sense to diminish the effect of the drug in the clonal expansion and the activation of the immune cells at the time of the immune stimulation.

Tumor growth was evaluated by caliper measurement of the tumor length and width 3 times per week. Tumor volume was calculated using the formula $\mathrm{V}=\left(\mathrm{d}^{2} \times \mathrm{D}\right) / 2$, where $\mathrm{V}$ is the volume in $\mathrm{mm}^{3}$, and $\mathrm{d}$ and $\mathrm{D}$ are the shortest and longest diameters, respectively.

\section{Sampling procedure}

Samples were obtained 21 days after tumor cell injection. All mice were sacrificed by cervical dislocation. Four mice per group were used to obtain samples of tumor, spleen and lymph nodes (inguinal lymph nodes on both sides) for flow cytometry analyses, and three mice per group to obtain the same samples for optical microscopy.

\section{Histological analysis}

Tumors and spleen were examined macroscopically and then fixed with buffered formalin solution and embedded in paraffin. Tissue sections $(4 \mu \mathrm{m})$ were stained with hematoxylin-eosin.

For the analysis of spleens, the results were expressed with a semiquantitative score system. One $(+)$, two $(++)$ or three $(+++)$ crosses refer to mild, moderate or severe hyperplasia in the red or white pulp, respectively. Similarly, for megakaryocytes, their marginal distributions were semi-quantified by considering one, two and three crosses for mild, moderate and severe increases, respectively.

For the tumors, histomorphometric determinations were performed on digitized photomicrographs of histological slides 


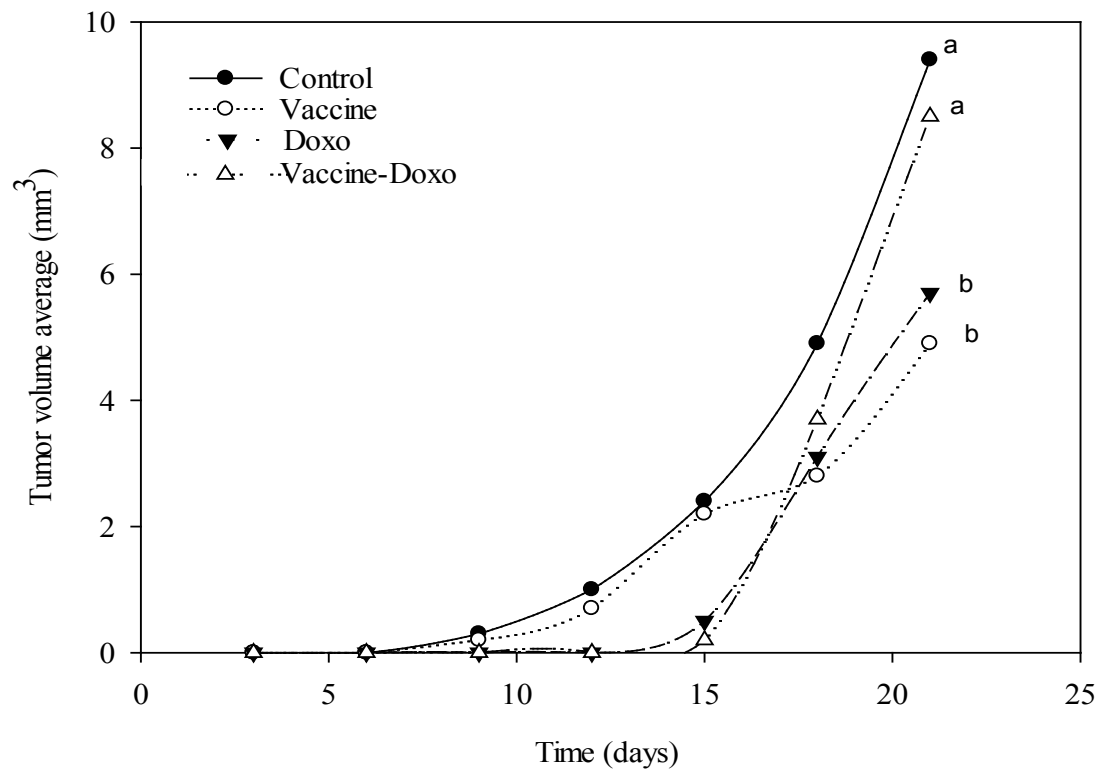

Figure 1: Rate of tumor growth. Results are expressed as the average of the tumor volume $\left(\mathrm{mm}^{3}\right)$ for each group. Each group consisted of 7 mice. Statistical analysis is show for the last measurement. Means values without a common letter differ significantly $(P<0.05)$.

Table 2: Hyperplasia and marginal megakaryocytosis in spleen.

\begin{tabular}{|c|c|c|c|c|c|}
\hline & & Control $^{\#}$ & Vaccine $^{\#}$ & Doxo" & Vaccine-Doxo \\
\hline \multirow[t]{3}{*}{$\operatorname{Red}_{\text {pulp }}{ }^{+}$} & + & $33 \%$ & $0 \%$ & $0 \%$ & $0 \%$ \\
\hline & ++ & $67 \%$ & $100 \%$ & $100 \%$ & $100 \%$ \\
\hline & +++ & $0 \%$ & $0 \%$ & $0 \%$ & $0 \%$ \\
\hline \multirow[t]{3}{*}{ White pulp ${ }^{\dagger}$} & + & $67 \%$ & $33 \%$ & $100 \%$ & $33 \%$ \\
\hline & ++ & $33 \%$ & $67 \%$ & $0 \%$ & $33 \%$ \\
\hline & +++ & $0 \%$ & $0 \%$ & $0 \%$ & $33 \%$ \\
\hline \multirow{3}{*}{$\begin{array}{l}\text { Marginal } \\
\text { megakaryocyte }^{\dagger \dagger}\end{array}$} & + & $33 \%$ & $0 \%$ & $0 \%$ & $33 \%$ \\
\hline & ++ & $33 \%$ & $100 \%$ & $67 \%$ & $67 \%$ \\
\hline & +++ & $33 \%$ & $0 \%$ & $33 \%$ & $0 \%$ \\
\hline
\end{tabular}

"Each column shows the percentage of mice from each group that presented de degree of histological alterations.

tHyperplasia of red and white pulp was qualitatively evaluated as slight $(+)$, moderate $(++)$ or severe $(+++)$.

${ }^{+}$The presence of marginal megakaryocytes was qualitatively expressed as slight $(+)$, moderate $(++)$ or severe $(+++)$.

using Image Proplus 4.5 Software. The parameters evaluated were: 1) Tumor necrosis area: Area total of necrosis and tumor necrosis per field (as observed at 100X). Measurements were performed on 5 randomly selected microscopic fields of tumor tissue. The criteria taken into account to measure areas were basophilic granular area excluding optically empty areas. 2) Number of mitosis: Ten randomly selected microscopic fields of tumor tissue were examined at 400X. The different mitosis phases (prophase, metaphase, anaphase and telophase) were considered. 3) Nuclear area: Ten randomly selected microscopic fields of tumor tissue (observed at 400X) were evaluated to measure the nuclear area of 30 randomly selected nuclei.

\section{Determination of CD11c (+) cells by flow cytometry}

Lymph nodes, spleen and tumors were collected, and mechanically disintegrated. For tumor samples this step was followed by an enzymatic digestion as was described above. Cell concentration was adjusted at $2 \pm 1 \times 10^{6}$ cells $/ \mathrm{ml}$. Isolated cells were incubated in darkness with the monoclonal FITC hamster anti-mouse CD11c antibody (BD Bioscience, San Diego, USA). Labeled cells were analysed with a FACS Partec Pas Cytometer (BD Bioscience, San Diego, CA, USA) equipped with Flomax software.

\section{Statistical analysis}

The design was a case-control study and the variables were studied qualitative and quantitatively. Sigma Stat V2.0 and SPSS v.15.0 were used. The comparisons were accomplished by an ANOVA general linear model followed by a Tukey's post-hoc test.

\section{Results}

\section{Effect of vaccination and doxorubicin treatment on tumor growth}

Tumor volume from mice of Vaccine group and Doxo group showed a statistically significant decrease $(\mathrm{P}<0.05)$ compared to the control group, at day 18. After 21 days, the Vaccine group showed an average decrease of $47 \%$ in tumor volume, and Doxo group $42 \%$ ( $\mathrm{P}<0.001)$, compared to the control animals. For the Vaccine-Doxo group no significant differences were observed when compared to the control group $(\mathrm{P}=0.182)$ (Figure 1); similarly, the treatment with low dose of doxorubicin $(30 \mu \mathrm{g})$ was not associated to significant changes compared to control group (data not shown).

\section{Histopathological modifications associated to vaccination and doxorubicin treatment in tumor bearing mice}

The analysis of spleens showed different levels of hyperplasia in the white and red pulp in the samples obtained from the different groups. In the control group, $67 \%$ of the samples showed moderate hyperplasia of the red and white pulp (Table 2). For marginal megakaryocytes, the $33 \%$ showed a slight amount, $33 \%$ moderate and the other 33\% severe amount (Table 2). The analysis of hyperplasia of red pulp in the different test groups showed the $100 \%$ of the samples with moderate hyperplasia. For the white pulp, the observations were different in the samples from the 3 test groups. For Vaccine group, $67 \%$ of the samples had moderate hyperplasia, for Doxo group the $100 \%$ of the samples showed slight hyperplasia, and for Vaccine-Doxo group, it was observed the same percentage (33.3\%) of samples with each score (Table 2). Finally, the evaluation of marginal megakaryocytes showed moderate amounts in the highest percentage of samples obtained from the 3 test groups (Table 2).

Microscopic appearance of adenocarcinoma M3 was analysed in control and test groups. For the control group all the samples presented adipocyte tissue and angio lymphatic invasion, and $67 \%$ of mice showed muscle and perivascular invasion (Table 3 ). This group also showed a high percentage (67\%) of severe tumor necrosis (Figure 2). The results obtained in the Vaccine and Doxo groups were similar, without perineural and angio lymphatic invasion, and with $100 \%$ of the samples showing perivascular invasion. However, in the Vaccine group, invasion of muscle tissue was observed, and for Doxo group the adipocyte tissue was invaded (Table 3). In the Vaccine-Doxo group predominance of adipocyte tissue, muscle and perivascular invasions were observed (Table 3 ). 
Table 3: Invasion of neoplastic cells

\begin{tabular}{|c|c|c|c|c|c|c|c|c|c|c|}
\hline & \multicolumn{2}{|c|}{ Adipocyte tissue } & \multicolumn{2}{|c|}{ Muscle tissue } & \multicolumn{2}{|c|}{ Perivascular } & \multicolumn{2}{|c|}{ Perineural } & \multicolumn{2}{|c|}{ Angiolymphatic } \\
\hline & Abs & Pres & Abs & Pres & Abs & Pres & Abs & Pres & Abs & Pres \\
\hline Control & 0 & 3 & 1 & 2 & 1 & 2 & 2 & 1 & 0 & 3 \\
\hline Vaccine & 3 & 0 & 0 & 3 & 0 & 3 & 3 & 0 & 3 & 0 \\
\hline Doxo & 0 & 3 & 3 & 0 & 0 & 3 & 3 & 0 & 3 & 0 \\
\hline Vaccine-Doxo & 0 & 3 & 0 & 3 & 2 & 1 & 3 & 0 & 3 & 0 \\
\hline
\end{tabular}

Each column shows the number of mice from each group (total $\mathrm{N}=3$ ) with or without the evaluated invasion. Abs and Pres mean absence and presence, respectively.

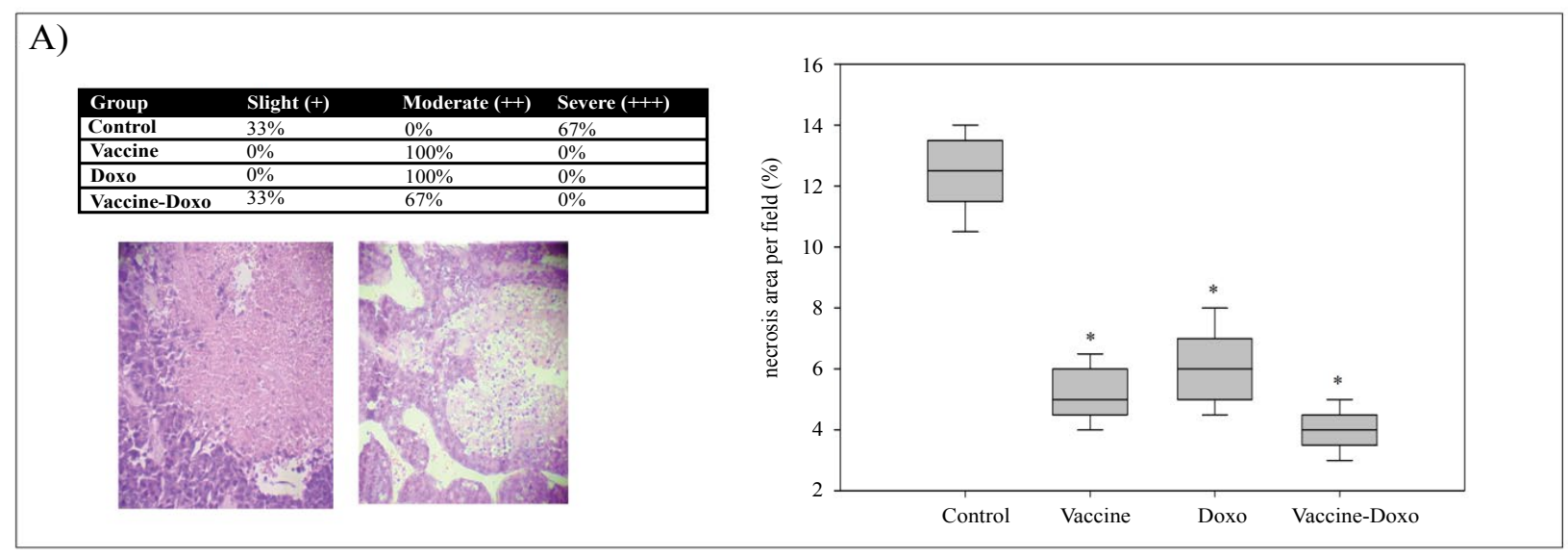

B)

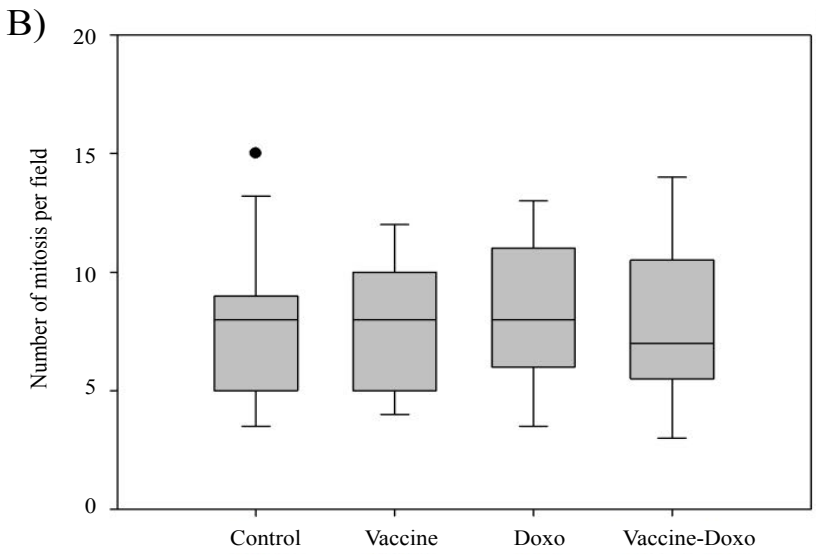

C)

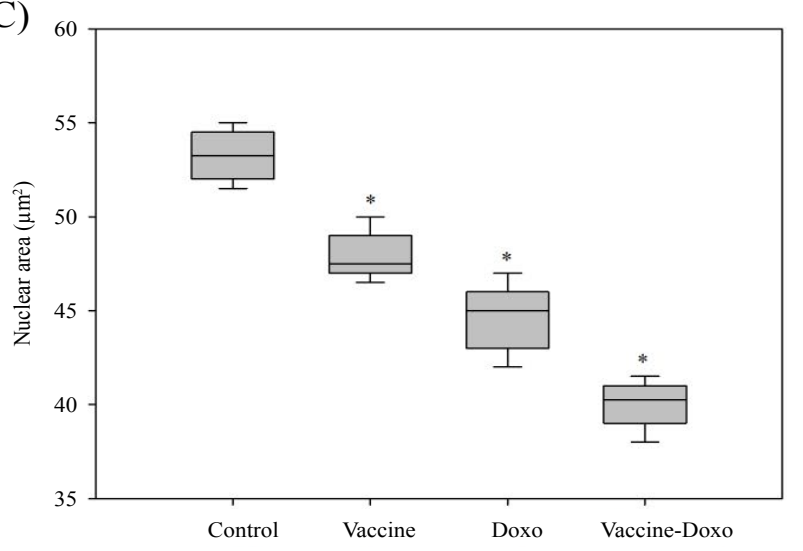

Figure 2: Histological evaluations of the tumors. Figure A shows the analysis of necrosis. The percentage of animals with slight, moderate or severe necrosis is showed in the table with representative microphotographs in the bottom (100X). The box plots show the percentage of necrosis area for each group. The number of mitosis counted per field $(400 \mathrm{X})$ and the nuclear area from 30 randomized nuclei are shown in figure $B$ and $C$, respectively. Significant differences $(P<0.05)$ compared to the Control group are represented with asterisk $\left(^{*}\right)$.

The histo metric analysis of the tumors is shown in (Figure 2). A significant decrease $(\mathrm{P}<0.001)$ in tumor necrosis area was observed with all the assayed treatments compared to the control group (Figure 2A). No significant differences between the control group and the test groups were observed for the mitosis index (Figure $2 \mathrm{~B})$. In contrast, a significant decrease $(\mathrm{P}<0.05)$ of nuclear area was observed when the test groups were compared with the control. All treatments decreased the nuclear area, with the largest diminution observed in the Vaccine-Doxo group (average of 25.5\% compared to the control (Figure 2C).

\section{Evaluation of dendritic cells}

The percentage of CD11c (+) cells in spleen showed a significant increase $(\mathrm{P}<0.001)$ in the Vaccine group, compared to the control group. A lower, but also significant increase $(\mathrm{P}<0.05)$ was observed in the Vaccine-Doxo group, compared to the control. No significant differences between the control group and the Doxo group $(\mathrm{P}=$ 0.150 ) were observed (Figure 3 ).

Lymph node did not show significant differences for the percentage of CD11c (+) between the control and test groups (Figure 3).

For tumor samples, even with large variation of data, the Vaccine group was the only one that showed a significant increase $(\mathrm{P}<0.05)$ in the percentage of $\mathrm{CD11c}(+)$ cells compared to the control group (Figure 3 ).

\section{Discussion}

The immune system is prepared to identify and specifically kill tumor cells, based on the expression of specific tumor antigens or certain molecules induced during tumor development [12]. However, although effector cells are present, they are often ineffective in vivo, and the tumor continues to grow and the metastasis can invade different organs. The sentinel lymph node is where the potentially auto-reactive lymphocytes encounter antigens and develop acquired immunity. The down-regulation of immune activity in the lymph nodes near the primary tumors indicates that sentinel nodes are more immuno modulated than the regional distant lymph nodes [13]. Also, it is known that immuno suppression is accentuated with chemotherapy treatments based on actively proliferating cell killing since immune cells are in this category, when activated, induce a response against foreign antigens [14].

These observations have led to the design of new therapeutic approaches for cancer. Traditional vaccines induce immunity to certain microbial antigens and prevent infectious diseases. Immunotherapy has proven to be effective in experimental animal tumor models and also in patients suffering cancer (as was described in the introduction of this article), with the Mycobacterium bovis BCG vaccine strain the most used. In the present work, the effectiveness of non-specific anti-tumor immunotherapy was demonstrated using a breast cancer 


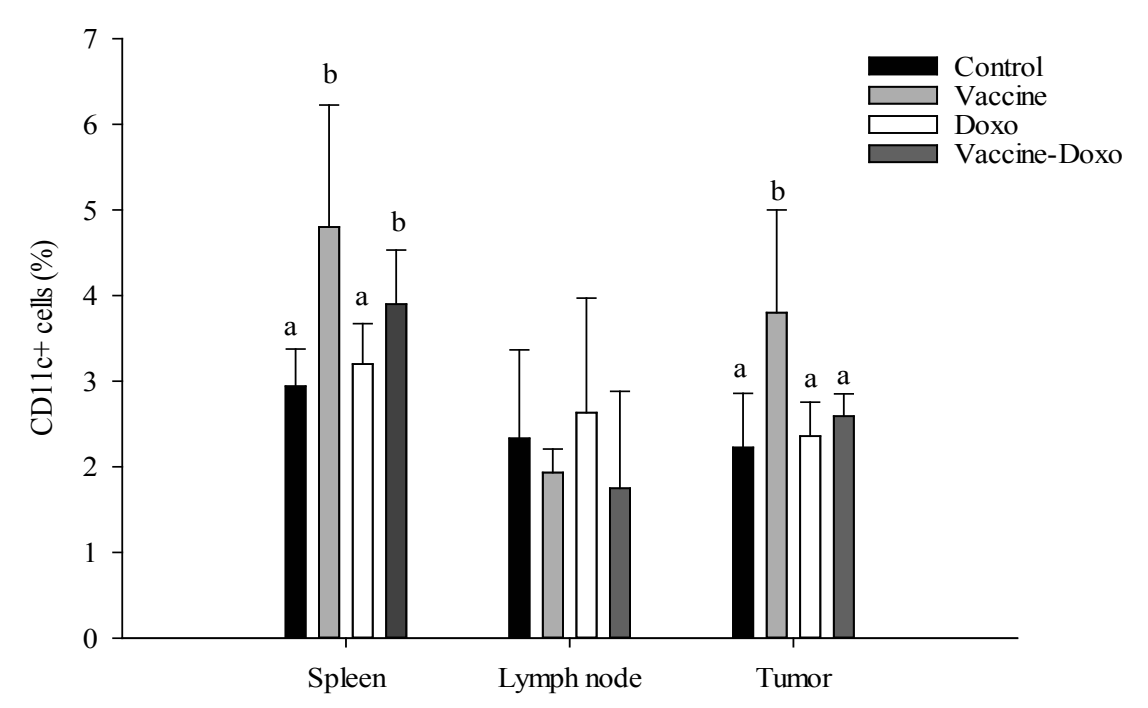

Figure 3: Presence of CD11c (+) cells. The percentage of CD11c (+) was evaluated in spleen, lymph node draining and tumor by flow cytometry. Results show the mean value \pm SD obtained for each group. For each sample, means without a common letter differ significantly $(P<0.05)$.

model in mice and a polymicrobial vaccine. Mice treated with the vaccine decreased tumor volume, similar to the animals treated with a cytostatic drug. These benefits were associated to histo morphometric modifications in the tumor tissues. The lower beneficial effects of the vaccine observed in the group that received it with the doxorubicin can be attributed to the immune suppressed effect of the drug, which interacts with the immune stimulation associated to the vaccine. The results obtained in the assayed model showed that when applied at the nearly same time with vaccinations, low dose of doxorubicin was capable of abrogating vaccine-mediated immune modulatory and antitumor effects, which was observed principally in the analysis of tumor volume. It is also important to note that the low dose of this chemotherapeutic drug was not associated to significant benefits compared to control group (data not shown).

The participation of immune cells in the response against the tumor was also evaluated at different levels. In the spleen, the vaccine group was the one that showed the highest hyperplasia of red and white pulp, and increased the marginal megakaryocytes. The benefit associated to this treatment was also analysed with the evaluation of invasion by tumor cells. This was similar in both vaccine and Doxo groups, and lower than in the control group. In addition, severe necrosis was not observed in the treated animals.

The presence of immune modulatory cytokines is an important aspect of the complex immune response to infection, and it has also a significant role in reducing tumor growth by non-specific vaccination, as was recently demonstrated for the intravesical administration of BCG in the treatment of bladder cancer [15]. Several cytokines have been detected in urine of the patients, such as IL-2, IL-8, IL-6, IL1ra, IL-10, IL-12[p70], IL-12[p40], TRAIL, and TNF- $\alpha$, and they can be used to predict the probability of tumor recurrence. These cytokines can be produced by the stimulated immune cells and some of them directly by the tumor cells. In this context, the study of dendritic cells (DC) has been gaining interest against cancer and they have also been evaluated in our experimental model. DC have a central role in developing specific immune response against different antigens an also in antitumor responses [16,17]. Two therapeutic approaches have been proven: the stimulation of in vivo development of protective immunity or by providing the patient with effector cells grown in vitro.

In the present work, we tried to stimulate in vivo an antitumor response and, in the respective model, to revert the immune suppression caused by chemotherapy, based on the cytokines induced by vaccination. The cytokines generated in the primary response and with re-stimulations (secondary response) could help in capturing the tumor antigens by the DC and promote antigen presentation and the development of specific immune response against the tumor.
A decrease in density of the DC in the sentinel node and in the para cortex area occupied by DC was observed in several solid tumors, such as in melanoma and breast cancer $[18,19]$. However, it is not clear whether the decreased density of DC in the sentinel node is due to a decreased flow of carriers DC antigen, or possibly to an increase in apoptosis of DC after interaction with $\mathrm{T}$ cells in the sentinel node [20].

CD11c is generally expressed by DC and it was used to analyse these cells in our model; however, this surface marker can express on other myeloid cells, lymphocytes and natural killer cells. The analysis of CD11c (+) cells showed that although the number was not increased in tumor draining nodes by the immune stimulating effect of the vaccine, this treatment increased the number of CD11c (+) cells in the spleen and tumor. This effect was related with decreased tumor volume in the vaccine-treated groups compared to the control group. In contrast, in the group treated with doxorubicin this correlation was not observed: the mice showed similar percentage of CD11c (+) cells in spleen and tumor than the ones of the control group. In these animals, the decrease of tumor volume would be due to the cytotoxic effect of the drug. Finally, in the group treated with both doxorubicin and vaccine, the tumor volume remained unchanged compared to the control although the percentage of CD11c (+) cells increased. These results indicate that there is not a synergistic anti-tumor effect between doxorubicin and the vaccine in the conditions assayed. The dose of doxurubicin(less than the one used in the group only treated with the cytotoxic agent) was not enough to affect the tumor growth, and even highlighting the positivity of increased CD11c (+) cells, probably the low doses of cytotoxic therapy affected induction or effector pathways of anti-tumor immune response. DC or other immune cells ( $\mathrm{T}$ helper lympocytes, cytotoxic lymphocytes, natural killer, Treg lymphocytes, among other) could be affected by the drug, and the correct immune response was not driven.

Our finding show that a non-specific vaccine used as immunotherapy prolonged an equilibrium phase in the tumor growth, similar to the doxorubicin treatment used in the model, although by different mechanisms. In this model, we did not observe tumor regression with the treatments assayed and future studies should be necessary by modifying the dose and/or type of cytotoxic drug to evaluate if a correct combination of vaccination and cytotoxic drug can be used for this purpose and to achieve increased activated DC in the tumor draining lymph nodes. The analysis of other cell markers, in addition to $\mathrm{CD} 11 \mathrm{c}$, will allow understanding the importance of DC or other immune cells in the beneficial effects obtained with the vaccination in our breast cancer model.

\section{Conflict of Interest}

The authors declare that they have no conflict of interest. 


\section{Acknowledgments}

This work was financially supported by Consejo de Investigación de la Universidad Nacional de Tucumán (CIUNT 26J/403), and Consejo Nacional de Investigaciones Científicas y Técnicas (CONICET), Argentina.

\section{References}

1. WHO (2015) Cancer: Fact sheet $\mathrm{N}^{\circ} 297$.

2. Chouaib S, El Hage F, Benlalam H, Mami-Chouaib F (2006) Immunotherapy of cancer: promise and reality. Med Sci 22: 755-759.

3. Emily A Rogena, Kenneth O Simbiri, G De Falco, L Leoncini, Leona Ayers et al. (2015) A review of the pattern of AIDS defining, HIV associated neoplasms and premalignant lesions diagnosed from 2000-2011 at Kenyatta National Hospital, Kenya. Infect Agent Cancer 10: 28.

4. Eralp Y, Wang X, Wang JP, Maughan MF, Polo JM, et al. (2004) Doxorubicin and paclitaxel enhance the antitumor efficacy of vaccines directed against HER 2/neu in a murine mammary carcinoma model. Breast Cancer Res 6 : R275-R283.

5. Coley WB (1991) The treatment of malignant tumors by repeated inoculations of erysipelas. With a report of ten original cases. 1893. Clin Orthop Rela $3-11$.

6. Zheng YQ, Naguib YW, Dong Y, Shi YC, Bou S, et al. (2015) Applications of bacillus Calmette-Guerin and recombinant bacillus Calmette-Guerin in vaccine development and tumor immunotherapy. Expert Rev Vaccines 14 1255-1275.

7. Patel SG, Cohen A, Weiner AB, Steinberg GD (2015) Intravesical therapy for bladder cancer. Expert Opin Pharmacother 16: 889-901.

8. Raffray L, Riviere P, Bonnet H, Duffau P, Longy-Boursier M (2015) Granulomatous hepatitis revealing a Mycobacterium bovis widespread infection following intravesical BCG therapy. Rev Med Interne 36: 626-630.

9. Marquez-Batalla S, Fraile-Villarejo E, Belhassen-Garcia M, GutierrezZubiaurre N, Cordero-Sanchez M (2014) Disseminated infection due to Mycobacterium bovis after intravesical BCG instillation. World J Clin Cases 2: 301-303.
10. Perez-Jacoiste Asin MA, Fernandez-Ruiz M, Lopez-Medrano F, Lumbreras C, Tejido A, et al. (2014) Bacillus Calmette-Guerin (BCG) infection following intravesical BCG administration as adjunctive therapy for bladder cancer: incidence, risk factors, and outcome in a single-institution series and review of the literature. Medicine (Baltimore) 93: 236-254.

11. Bal de Kier Joffe E, Puricelli L, Vidal MCC, Sacerdote de Lustig E (1983) Characterization of two murine mammary deno carcinoma tumors with different metastatic ability. J Exp Clin Cancer Res 2: 151-160.

12. Vesely MD, Kershaw MH, Schreiber RD, Smyth MJ (2011) Natural innate and adaptive immunity to cancer. Annu Rev Immunol 29: 235-271.

13. Nakamura S, Yaguchi T, Kawamura N, Kobayashi A, Sakurai T, et al. (2014) TGF-beta1 in tumor microenvironments induces immuno suppression in the tumors and sentinel lymph nodes and promotes tumor progression. $J$ Immunother 37: 63-72.

14. Rasmussen L, Arvin A (1982) Chemotherapy-induced immuno suppression. Environ Health Perspect 43: 21-25.

15. Kamat AM, Briggman J, Urbauer DL, Svatek R, Nogueras Gonzalez GM et al. (2015) Cytokine Panel for Response to Intravesical Therapy (CyPRIT): Nomogram of Changes in Urinary Cytokine Levels Predicts Patient Response to Bacillus Calmette-Guerin. Eur Urol 69: 197-200.

16. Kawano M, Tanaka K, Itonaga I, Iwasaki T, Miyazaki M et al. (2015) Dendritic cells combined with anti-GITR antibody produce antitumor effects in osteosarcoma. Oncol Rep 34: 1995-2001.

17. Pardee AD, Yano H, Weinstein AM, Ponce AA, Ethridge AD et al. (2015) Route of antigen delivery impacts the immunostimulatory activity of dendritic cell-based vaccines for hepatocellular carcinoma. J Immunother Cancer 3: 32.

18. Botella-Estrada R, Dasi F, Ramos D, Nagore E, Herrero MJ et al. (2005) Cytokine expression and dendritic cell density in melanoma sentinel nodes. Melanoma Res 15: 99-106.

19. Poindexter NJ, Sahin A, Hunt KK, Grimm EA (2004) Analysis of dendritic cells in tumor-free and tumor-containing sentinel lymph nodes from patients with breast cancer. Breast Cancer Res 6: R408-R415.

20. Cochran AJ, Huang RR, Su A, Itakura E, Wen DR (2015) Is sentinel node susceptibility to metastases related to nodal immune modulation? Cancer $\mathrm{J}$ 21: $39-46$. 